\title{
ЭЛЕКТРОМАГНИТНАЯ СУШКА ВЛАЖНЫХ МАТЕРИАЛОВ С МАЛОЙ ГЛУБИНОЙ ПРОНИКНОВЕНИЯ СВЧ-ИЗЛУЧЕНИЯ В УСЛОВИЯХ ТЕПЛОСБРОСА РАДИАЦИЕЙ И КОНВЕКЦИЕЙ. III. СТАДИЯ ПАДАЮЩЕЙ СКОРОСТИ СУШКИ
}

\author{
Саломатов Владимир Васильевич1,2, \\ salomatov.vv@mail.ru \\ Карелин Вадим Александрович1,2, \\ vad2hen@mail.ru
}

\author{
Саломатов Василий Владимирович2, \\ vvsalomatov@mail.ru \\ 1 Новосибирский государственный университет, \\ Россия, 630090, г. Новосибирск, ул. Пирогова, 1. \\ 2 Институт теплофизики им. С.С. Кутателадзе СО РАН, \\ Россия, 630090, г. Новосибирск, пр. Академика Лаврентьева, 1.
}

\begin{abstract}
Актуальность исследования продиктована необходимостью разработки математических моделей СВЧ-нагрева и МВ-сушки влажных материалов для получения технологически оптимальных и экономически выгодных режимов. Настоящая публикация является продолжением статей тех же авторов в «Известия Томского политехнического университета. Инжиниринг георесурсов», в которой с помощью математического моделирования подробно был изучен процесс на первой и второй стадиях сушки - стадии прогрева, когда теплообмен между поверхностью влажного тела и окружающей средой осуществляется за счет радиации и конвекции, а СВЧ-энергия поглощается поверхностным слоем из-за малой её глубины проникновения, а также стадии постоянной скорости сушки. Построены асимптотические решения этой нелинейной задачи для мальх и больших значений безразмерного времени, востребованные инженерной практикой, как для параметрического анализа, так и для проведения оперативных расчетов. Подход, связанньй с рассмотрением третьей стадии - стадии падающей скорости сушки, основан на установлении базового уравнения сушки, которое обеспечивает взаимосвязь между теплообменом и влагообменом с помощью критерия Ребиндера.

Цель: постановка задачи третьего этапа СВЧ-сушки влажного материала - этапа падающей скорости сушки - и реализация теоретического решения по определению распределения температурного поля по толщине слоя и величины скорости сушки.

Объектом исследования является плоский слой влажного материала - уголь, песок, древесина и др. капиллярно-пористье массивы, на которые воздействует СВЧ-излучение. Такие материалы обладают высокой диэлектрической проницаемостью и как следствие весьма эфффективно поглощают СВЧ-излучение, которое почти на 100 \% преобразуется в тепловую энергию.

Методы исследования связаны с математическим моделированием, в основе которых лежат уравнения электродинамики Максвелла и тепловлагопереноса А.В. Лькова. В данной статье при оценке параметров тепловлагопереноса учет теплообмена ведется более детально, нежели массообмена. Также одной из особенностей данной задачи электромагнитной сушки является рассмотрение материалов с малой глубиной поглощения, в силу чего в системе уравнений для нагрева источниковый член находится в граничном условии.

В результате исследований были определены температура и влагосодержание подсушиваемого тела в режиме падающей скорости сушки, получены расчетные соотношения аналитического характера для малых и больших параметров времени.
\end{abstract}

\section{Ключевые слова:}

СВЧ-излучение, электромагнитная сушка, капиллярно-пористый массив, теплоизлучение, конвекция, уравнение тепловлагопереноса А.В. Лыкова, стадия падающей скорости сушки.

\section{Введение}

Известно, что СВЧ-излучение представляет собой электромагнитные волны с частотой от 300 МГц до 30 ГГц, с длиной волны от 1 мм до 1 м. Практически до недавнего времени использование микроволновой энергии как в промышленности, так и в быту было ограничено из-за дороговизны СВЧ-техники [1-4]. Усовершенствование технологий производства микроволновых приборов привело к широкому распространению устройств на основе СВЧ-энергии, в качестве которых выступают микроволновые печи, спутниковые антенны, сотовые смартфоны и др. Особо востребовано СВЧ-излучение для генерации тепла и электромагнитной сушки [5-12]. Физика нагрева микроволнами связана, прежде всего, с воздействием электромагнитного излучения на полярные молекулы, главным образом воды, которые под воздействием высокочастотного внешнего электрического поля начинают активно колебаться, затрагивая соседние молекулы. В результате чего происходит регулируемый диэлектрический нагрев, температура материала повышается. За счет фазового перехода «вода-пар» идет обезвоживание, возникают градиенты температуры и влагосодержания, направления которых совпадают, что интенсифицирует сушку $[13,14]$.

Исключительная роль в процессе электромагнитной сушки принадлежит такому свойству материала, как диэлектрическая проницаемость. Главная особенность поведения данной характеристики заключается в том, что её максимум достигается при частотах, 
соответствующих СВЧ-диапазону. Изменение уровня диэлектрической проницаемости сильнейшим образом зависит от влажности. Его величина составляет для сухих материалов порядка нескольких единиц, а с повышенной влажностью - порядка сотни единиц. Такое поведение диэлектрической проницаемости не только обеспечивает удаление влаги из продукта, но и выравнивает влажность по объему [15-18]. На данный момент с помощью микроволнового излучения решаются актуальные задачи в таких отраслях промышленности, как химическая, строительная, деревообрабатывающая, энергетическая и др. В области производства стройматериалов СВЧ-технологии применяются при изготовлении кирпича, а также в процессах сушки древесины, лекарственных растений и т. д.

Перейдем к рассмотрению заключительной стадии сушки - стадии падающей скорости. Важно отметить, в периоде падающей скорости интенсивность теплообмена $q_{n}$ и интенсивность сушки $j_{n}$ непрерывно уменьшаются по времени. Важнейшими критериями для этой стадии выступают теплообменный и массообменный критерии Кирпичева $[19,20]$

$$
K i_{q}(t)=\frac{q_{n}(t) l}{\lambda T_{c}}, K i_{m}=\frac{j_{n}(t) l}{a_{m} \rho_{0} u_{0}} .
$$

Между $K i_{q}(t)$ и $K i_{m}(m)$ для данной стадии существует связь в следующей критериальной форме

$$
K i_{q}(t)=K i_{m}(t) L u K o(1+R b),
$$

где $R b=\frac{c}{r}\left(\frac{d T}{d u}\right)-$ критерий Ребиндера.

Ниже будут построены асимптотические решения нелинейной задачи тепло-влагопереноса для малых и больших безразмерных времен Fo. При этом используется следующее свойство интегрального преобразования Лапаса. Большим значениям параметра преобразования Лапласа s соответствуют малые значения Fo, и наоборот - малым значениям параметра преобразования s соответствуют большие значения Fo.

Задача периода падающей скорости сушки для изменений полей безразмерной температуры и влагосодержания выглядит следующим образом:

$$
\begin{gathered}
\frac{\partial \Theta(X, F o)}{\partial F o}-(1+\varepsilon K o P n L u) \frac{\partial \Theta^{2}(X, F o)}{\partial X^{2}}- \\
-\varepsilon K o L u \frac{\partial U^{2}(X, F o)}{\partial X^{2}}=0, \\
\frac{\partial U(X, F o)}{\partial F o}=L u \frac{\partial^{2} U(X, F o)}{\partial X^{2}}-P n L u \frac{\partial^{2} \Theta(X, F o)}{\partial X^{2}}, \\
\frac{\partial \Theta(1, F o)}{\partial X}+K i_{q}(F o)-(1-\varepsilon) L u K o K i_{m}(F o)=0, \\
-\frac{\partial U(1, F o)}{\partial X}+P n \frac{\partial \Theta(1, F o)}{\partial X}+K i_{m}(F o)=0, \\
\frac{\partial \Theta(0, F o)}{\partial X}=0 ; \frac{\partial U(0, F o)}{\partial X}=0 ; \\
\Theta(X, 0)=\bar{\Theta}_{0} ; U(X, 0)=\bar{U}_{0},
\end{gathered}
$$

где введены следующие критерии тепловлагопереноса:
$L u=\frac{a_{m}}{a}-$ критерий Лыкова;

Ko $=\frac{r \Delta u}{c \Delta T}-$ критерий Коссовича;

$P n=\frac{\delta \Delta T}{\Delta u}-$ критерий Поснова;

$R b=\frac{c b}{r}-$ критерий Ребиндера;

$K i_{q}=\frac{q_{n} R_{v}}{\lambda T_{c}}-$ теплообменный критерий Кирпичева;

$K i_{m}=\frac{j_{n} R_{v}}{a_{m} \rho_{0} u_{0}}-$ массообменный критерий Кирпичева.

\section{Асимптотика для малых чисел Фурье \\ (больших значениях параметра преобразования Лапласа s)}

Представим решение задачи в изображениях (1)-(6) через передаточные функции:

$$
\begin{gathered}
\Theta_{L}(X, s)-\frac{\bar{\Theta}_{0}}{s} \simeq K i_{q L}(s) P_{1}(X, s)+K i_{m L}(s) P_{2}(X, s) ; \\
U_{L}(X, s)-\frac{\bar{U}_{0}}{s} \simeq K i_{q L}(s) M_{1}(X, s)+K i_{m L}(s) M_{2}(X, s),(7)
\end{gathered}
$$

где $P_{1}, P_{2}, M_{1}, M_{2}-$ передаточные функции. Представим их в виде разложения по большому параметру $s$ в виде:

$$
\begin{aligned}
& P_{1}(X, s) \simeq \varphi_{0}(X, s) \frac{e^{-\sqrt{s}}}{\sqrt{s}}+\varphi_{1}(X, s) \frac{e^{-2 \sqrt{s}}}{s}+\ldots \\
& P_{2}(X, s) \simeq \psi_{0}(X, s) \frac{e^{-\sqrt{s}}}{\sqrt{s}}+\psi_{1}(X, s) \frac{e^{-2 \sqrt{s}}}{s}+\ldots \\
& M_{1}(X, s) \simeq \chi_{0}(X, s) \frac{e^{-\sqrt{s}}}{\sqrt{s}}+\chi_{1}(X, s) \frac{e^{-2 \sqrt{s}}}{s}+\ldots \\
& M_{2}(X, s) \simeq v_{0}(X, s) \frac{e^{-\sqrt{s}}}{\sqrt{s}}+v_{1}(X, s) \frac{e^{-2 \sqrt{s}}}{s}+\ldots
\end{aligned}
$$

Также введем обозначения:

$$
\begin{gathered}
Y_{1}=1+\varepsilon K o P n L u ; \\
Y_{2}=-\varepsilon K o L u .
\end{gathered}
$$

Представим (1) в изображениях по Лапласу:

$$
s\left(\Theta_{L}-\frac{\bar{\Theta}_{0}}{s}\right)=Y_{1} \Theta_{L}^{\prime \prime}+Y_{2} U_{L}^{\prime \prime} .
$$

Найдем $\Theta_{L}^{\prime \prime}, U_{L}^{\prime \prime}$ :

Дифференцируя (7), имеем:

$$
\begin{aligned}
\Theta_{L}^{\prime \prime} & \simeq K i_{q L}(s)\left[\varphi_{0}^{\prime \prime}(X, s) \frac{e^{-\sqrt{s}}}{\sqrt{s}}+\varphi_{1}^{\prime \prime}(X, s) \frac{e^{-2 \sqrt{s}}}{s}+\ldots\right]+ \\
+ & K i_{m L}(s)\left[\psi_{0}^{\prime \prime}(X, s) \frac{e^{-\sqrt{s}}}{\sqrt{s}}+\psi_{1}^{\prime \prime}(X, s) \frac{e^{-2 \sqrt{s}}}{s}+\ldots\right] \\
U_{L}^{\prime \prime} & \simeq K i_{q L}(s)\left[\chi_{0}^{\prime \prime}(X, s) \frac{e^{-\sqrt{s}}}{\sqrt{s}}+\chi_{1}^{\prime \prime}(X, s) \frac{e^{-2 \sqrt{s}}}{s}+\ldots\right]+ \\
+ & K i_{m L}(s)\left[v_{0}^{\prime \prime}(X, s) \frac{e^{-\sqrt{s}}}{\sqrt{s}}+v_{1}^{\prime \prime}(X, s) \frac{e^{-2 \sqrt{s}}}{s}+\ldots\right]
\end{aligned}
$$


Подставляя (10) в (9), запишем:

$$
\begin{aligned}
& s\left[\begin{array}{l}
K i_{q L}(s)\left(\varphi_{0}(X, s) \frac{e^{-\sqrt{s}}}{\sqrt{s}}+\varphi_{1}(X, s) \frac{e^{-2 \sqrt{s}}}{s}+\ldots\right)+ \\
+K i_{m L}(s)\left(\psi_{0}(X, s) \frac{e^{-\sqrt{s}}}{\sqrt{s}}+\psi_{1}(X, s) \frac{e^{-2 \sqrt{s}}}{s}+\ldots\right)
\end{array}\right]= \\
& =Y_{1}\left[\begin{array}{l}
K i_{q L}(s)\left[\varphi_{0}^{\prime \prime}(X, s) \frac{e^{-\sqrt{s}}}{\sqrt{s}}+\varphi_{1}^{\prime \prime}(X, s) \frac{e^{-2 \sqrt{s}}}{s}+\ldots\right]+ \\
+K i_{m L}(s)\left[\psi_{0}^{\prime \prime}(X, s) \frac{e^{-\sqrt{s}}}{\sqrt{s}}+\psi_{1}^{\prime \prime}(X, s) \frac{e^{-2 \sqrt{s}}}{s}+\ldots\right]
\end{array}\right]+ \\
& +Y_{2}\left[\begin{array}{l}
K i_{q L}(s)\left[\chi_{0}^{\prime \prime}(X, s) \frac{e^{-\sqrt{s}}}{\sqrt{s}}+\chi_{1}^{\prime \prime}(X, s) \frac{e^{-2 \sqrt{s}}}{s}+\ldots\right]+ \\
+K i_{m L}(s)\left[v_{0}^{\prime \prime}(X, s) \frac{e^{-\sqrt{s}}}{\sqrt{s}}+v_{1}^{\prime \prime}(X, s) \frac{e^{-2 \sqrt{s}}}{s}+\ldots\right]
\end{array}\right] .
\end{aligned}
$$

Группируя члены, получим следующие уравнения: Слагаемые при $K i_{q L}(s) \frac{e^{-\sqrt{s}}}{\sqrt{s}}: \quad s \varphi_{0}=Y_{1} \varphi_{0}^{\prime \prime}+Y_{2} \chi_{0}^{\prime \prime}$;

Слагаемые при $K i_{q L}(s) \frac{e^{-2 \sqrt{s}}}{s}: \quad s \varphi_{1}=Y_{1} \varphi_{1}^{\prime \prime}+Y_{2} \chi_{1}^{\prime \prime}$;

Слагаемые при $K i_{m L}(s) \frac{e^{-\sqrt{s}}}{\sqrt{s}}: s \psi_{0}=Y_{1} \psi_{0}^{\prime \prime}+Y_{2} v_{0}^{\prime \prime}$;

Слагаемые при $K i_{m L}(s) \frac{e^{-2 \sqrt{s}}}{s}: s \psi_{1}=Y_{1} \psi_{1}^{\prime \prime}+Y_{2} v_{1}^{\prime \prime}$.

Для первого члена разложения можно записать систему:

$$
\begin{gathered}
s \varphi_{0}=Y_{1} \varphi_{0}^{\prime \prime} ; \\
\varphi_{0}^{\prime}(0, s)=0 ; \\
\varphi_{0}^{\prime}(1, s)=-\frac{\sqrt{s}}{e^{-\sqrt{s}}} .
\end{gathered}
$$

Решая систему (11), имеем (12):

$$
\varphi_{0}(X, s)=-\frac{e^{\sqrt{s}+\sqrt{\frac{s}{Y_{1}}}-\sqrt{\frac{s}{Y_{1}}} X\left(1+e^{2 \sqrt{\frac{s}{Y_{1}}} X}\right) \sqrt{Y_{1}}}}{-1+e^{2 \sqrt{\frac{s}{Y_{1}}}}} .
$$

Аналогично для $\psi_{0}$ получаем систему (13):

$$
\begin{gathered}
s \psi_{0}=Y_{1} \psi_{0}^{\prime \prime} \\
\psi_{0}^{\prime}(0, s)=0 \\
\psi_{0}^{\prime}(1, s)=\frac{\sqrt{s}}{e^{-\sqrt{s}}}(1-\varepsilon) \text { LuKo }
\end{gathered}
$$

решением которой является (14):

$$
\psi_{0}(X, s)=(1-\varepsilon) L u K o \frac{e^{\sqrt{s}+\sqrt{\frac{s}{Y_{1}}}-\sqrt{\frac{s}{Y_{1}}} X\left(1+e^{2 \sqrt{\frac{s}{Y_{1}}} X}\right) \sqrt{Y_{1}}}}{-1+e^{\sqrt[2]{\frac{s}{Y_{1}}}}} .
$$

В итоге с учетом первого члена разложения для изображения температуры и связь $K i_{q L}$ и $K i_{m L}$ имеем:

$$
\begin{aligned}
& \Theta_{L}(X, s)-\frac{\bar{\Theta}_{0}}{s} \simeq K i_{q L}(s)\left(-\frac{e^{\sqrt{\frac{s}{Y_{1}}}-\sqrt{\frac{s}{Y_{1}}} X\left(1+e^{2 \sqrt{\frac{s}{Y_{1}}}}\right) \sqrt{Y_{1}}}}{\left(-1+e^{2 \sqrt{\frac{s}{Y_{1}}}}\right) \sqrt{s}}\right)+ \\
& +K i_{m L}(s)\left((1-\varepsilon) L u K o \frac{e^{\sqrt{\frac{s}{Y_{1}}}-\sqrt{\frac{s}{Y_{1}}} X\left(1+e^{\left.2 \sqrt{\frac{s}{Y_{1}}} X\right)}\right) \sqrt{Y_{1}}}}{\left(-1+e^{2 \sqrt{\frac{s}{Y_{1}}}}\right) \sqrt{s}}\right)= \\
& =\left(\frac{e^{\sqrt{\frac{s}{Y_{1}}}-\sqrt{\frac{s}{Y_{1}}} X\left(1+e^{2 \sqrt{\frac{s}{Y_{1}}}}\right) \sqrt{Y_{1}}}}{\left(-1+e^{2 \sqrt{\frac{s}{Y_{1}}}}\right) \sqrt{S}}\right) \times \\
& \times\left[-K i_{q L}(s)+K i_{m L}(s)(1-\varepsilon) L u K o\right]= \\
& =\left(\frac{e^{\sqrt{\frac{s}{Y_{1}}}-\sqrt{\frac{s}{Y_{1}}} X\left(1+e^{2 \sqrt{\frac{s}{Y_{1}}} X}\right) \sqrt{Y_{1}}}}{\left(-1+e^{2 \sqrt{\frac{s}{Y_{1}}}}\right) \sqrt{s}}\right)\left[-K i_{q L}(s)+K i_{q L}(s) \frac{1-\varepsilon}{1+R b}\right]= \\
& =K i_{q L}\left(-1+\frac{1-\varepsilon}{1+R b}\right)\left(\frac{e^{\sqrt{\frac{s}{Y_{1}}}-\sqrt{\frac{s}{Y_{1}}} X}\left(1+e^{2 \sqrt{\frac{s}{Y_{1}}}}\right) \sqrt{Y_{1}}}{\left(-1+e^{2 \sqrt{\frac{s}{Y_{1}}}}\right) \sqrt{s}} .\right.
\end{aligned}
$$

Данное решение можно представить в следующем виде:

$$
\begin{gathered}
\Theta_{L}(X, s)-\frac{\bar{\Theta}_{0}}{s} \simeq \\
\simeq K i_{q L}\left(-1+\frac{1-\varepsilon}{1+R b}\right) \frac{\left(e^{-\sqrt{\frac{s}{Y_{1}}} X}+e^{\sqrt{\frac{s}{Y_{1}}} X}\right) \sqrt{Y_{1}}}{\left(-e^{-\sqrt{\frac{s}{Y_{1}}}}+e^{\sqrt{\frac{s}{Y_{1}}}}\right) \sqrt{s}}= \\
=K i_{q L}\left(-1+\frac{1-\varepsilon}{1+R b}\right) \frac{\operatorname{ch}\left(\sqrt{\frac{s}{Y_{1}}} X\right)}{\sqrt{\frac{s}{Y_{1}}} \cdot \operatorname{sh}\left(\sqrt{\frac{s}{Y_{1}}}\right)} .
\end{gathered}
$$

При больших $s$ :

$$
\frac{1}{\operatorname{sh}\left(\sqrt{\frac{s}{Y_{1}}}\right)} \approx 2 e^{-\sqrt{\frac{s}{Y_{1}}}}
$$

$\Theta_{L}(X, s)-\frac{\bar{\Theta}_{0}}{s} \simeq \mathrm{Ki}_{q L}\left(-1+\frac{1-\varepsilon}{1+R b}\right) \frac{\operatorname{ch}\left(\sqrt{\frac{s}{Y_{1}}} X\right)}{\sqrt{\frac{s}{Y_{1}}}} 2 e^{-\sqrt{\frac{s}{Y_{1}}}}$. 
Найдем обратное преобразование Лапласа от $\frac{\operatorname{ch}\left(\sqrt{\frac{s}{Y_{1}}} X\right)}{\sqrt{\frac{s}{Y_{1}}}} 2 e^{-\sqrt{\frac{s}{Y_{1}}}}:$

$L^{-1}\left(\frac{\operatorname{ch}\left(\sqrt{\frac{s}{Y_{1}}} X\right)}{\sqrt{\frac{s}{Y_{1}}}} 2 e^{-\sqrt{\frac{s}{Y_{1}}}}\right)=L^{-1}\left(\frac{e^{\sqrt{\frac{s}{Y_{1}}} X}+e^{-\sqrt{\frac{s}{Y_{1}}} X}}{\sqrt{\frac{s}{Y_{1}}}} e^{-\sqrt{\frac{s}{Y_{1}}}}\right)=$ $=L^{-1}\left(\frac{e^{\sqrt{\frac{S}{Y_{1}}}(X-1)}+e^{-\sqrt{\frac{S}{Y_{1}}(X+1)}}}{\sqrt{\frac{S}{Y_{1}}}}\right)=\left(e^{-\frac{(1-X)^{2}}{4 F O Y_{1}}}+e^{-\frac{(X+1)^{2}}{4 F o Y_{1}}}\right) \sqrt{\frac{Y_{1}}{\pi F o}}$.

Для нахождения оригинала от (15) воспользуемся теоремой о свертке:

$$
\begin{gathered}
\Theta(X, F o) \approx\left(1+\frac{1-\varepsilon}{1+R b}\right) \sqrt{Y_{1}} \int_{0}^{F o} K i_{q}(\eta) \times \\
\times\left[\left(e^{-\frac{(1-X)^{2}}{4(F o-\eta) Y_{1}}}+e^{-\frac{(X+1)^{2}}{4(F o-\eta) Y_{1}}}\right) \frac{1}{\sqrt{\pi(F o-\eta)}}\right] d \eta+\ldots \ldots .
\end{gathered}
$$

Для раскрытия интеграла разложим $K i_{q}(\eta)$ в ряд Тейлора:

$$
K i_{q}(\eta) \approx K i_{q}(F o)+(\eta-F o) K i_{q}^{\prime}(F o)+\ldots
$$

Также учтем:

$$
K i_{q}(\eta)=K i_{w}-S k\left[\Theta_{w}{ }^{4}-1\right]-B i\left[\Theta_{w}-1\right] .
$$

В результате получим

$\Theta(X, F o) \approx\left(1+\frac{1-\varepsilon}{1+R b}\right)\left(K i_{w}-S k\left[\Theta_{w}{ }^{4}-1\right]-B i\left[\Theta_{w}-1\right]\right) \times$ $\times \sqrt{Y_{1}} \int_{0}^{F o}\left[\left(e^{-\frac{(1-X)^{2}}{4(F o-\eta) Y_{1}}}+e^{-\frac{(X+1)^{2}}{4(F o-\eta) Y_{1}}}\right) \frac{1}{\sqrt{\pi(F o-\eta)}}\right] d \eta+\ldots \ldots \ldots$

Проводя дальнейшее интегрирование, запишем температурную асимптотику для малых чисел Фурье с учетом первого члена разложения

$$
\begin{aligned}
\Theta(X, F o) & \approx\left(1+\frac{1-\varepsilon}{1+R b}\right) \frac{\left(K i_{w}-S k\left[\Theta_{w}{ }^{4}-1\right]-B i\left[\Theta_{w}-1\right]\right)}{24 Y_{1}^{\frac{1}{4}}} \times \\
\times & {\left.\left[\begin{array}{l}
\left.(1+X)^{3} \operatorname{erfc}\left(\frac{(1+X) \sqrt{F_{0}}}{2 \sqrt{Y_{1}}}\right)+\right] \\
+(1-X)^{3} \operatorname{erfc}\left(\frac{(1-X) \sqrt{F_{o}}}{2 \sqrt{Y_{1}}}\right)
\end{array}\right)\right]+\ldots \ldots \ldots . }
\end{aligned}
$$

Подставляя $X=1$, получим уравнение четвертой степени для поверхностной температуры (16):

$$
\begin{gathered}
\Theta_{w} \approx\left(1+\frac{1-\varepsilon}{1+R b}\right) \times \\
\times \frac{\left(K i_{w}-S k\left[\Theta_{w}{ }^{4}-1\right]-B i\left[\Theta_{w}-1\right]\right)}{3 Y_{1}^{\frac{1}{4}}} \operatorname{erf}\left(\frac{\sqrt{F_{0}}}{\sqrt{Y_{1}}}\right) .
\end{gathered}
$$

В итоге действительный положительный корень данного уравнения можно записать так

$$
\Theta_{w} \approx \frac{1}{2} \sqrt{\frac{4\left(\frac{2}{3}\right)^{\frac{1}{3}} D}{F}+\frac{F}{2^{\frac{1}{3}} 3^{\frac{2}{3}} B}}-
$$

$$
\begin{gathered}
-\frac{1}{2} \sqrt{-\frac{4\left(\frac{2}{3}\right)^{\frac{1}{3}} D}{F}-\frac{F}{2^{\frac{1}{3}} 3^{\frac{2}{3}} B}-\frac{2 C}{B \sqrt{\frac{4\left(\frac{2}{3}\right)^{\frac{1}{3}} D}{F}+\frac{F}{2^{\frac{1}{3}} 3^{\frac{2}{3}} B}}}} ; \\
F=\left(9 B C^{2}+\sqrt{3} \sqrt{27 B^{2} C^{4}-256 B^{3} D^{3}}\right)^{\frac{1}{3}} ; \\
D=-A-B-C+1 ; \\
C=A_{0} B i\left(1-2 \sqrt{\frac{F o}{\pi Y_{1}}}\right)+1 ; \\
B=A_{0} S k\left(1-2 \sqrt{\frac{F o}{\pi Y_{1}}}\right) ; \\
A=A_{0} K i_{w}\left(1-2 \sqrt{\frac{F o}{\pi Y_{1}}}\right) ; \\
A_{0}=\frac{\left(1+\frac{1-\varepsilon}{1+R b}\right)}{3 Y_{1}^{\frac{1}{4}}} .
\end{gathered}
$$

Зная граничную температуру, окончательно можно определить температурное распределение для малых чисел Фурье с учетом первого члена разложения:

$$
\begin{aligned}
\Theta(X, F o) \approx\left(1+\frac{1-\varepsilon}{1+R b}\right) \frac{\left(K i_{w}-S k\left[\Theta_{w}{ }^{4}-1\right]-B i\left[\Theta_{w}-1\right]\right)}{24 Y_{1}^{\frac{1}{4}}} \times \\
\times\left[\begin{array}{l}
\left.(1+X)^{3} \operatorname{erfc}\left(\frac{(1+X) \sqrt{F_{o}}}{2 \sqrt{Y_{1}}}\right)+\right] \\
\left.+(1-X)^{3} \operatorname{erfc}\left(\frac{(1-X) \sqrt{F_{o}}}{2 \sqrt{Y_{1}}}\right)\right]+\ldots \ldots
\end{array}\right.
\end{aligned}
$$

\section{Асимптотика при больших Fo (малые значения} параметра преобразования Лапласа s)

Представим решение $\Theta_{L}(X, s)$ через передаточные функции $P_{1}$ и $P_{2}(17)$

$$
\Theta_{L}(X, s)=K i_{q L}(s) P_{1}(X, s)+K i_{m L}(s) P_{2}(X, s),
$$

которые разложим в ряд по малому параметру $s$ (18), (19)

$$
\begin{aligned}
& P_{1}(X, s) \simeq \varphi_{0}(X, s)+s \varphi_{1}(X, s)+s^{2} \varphi_{2}(X, s)+\ldots \\
& P_{2}(X, s) \simeq \psi_{0}(X, s)+s \psi_{1}(X, s)+s^{2} \psi_{2}(X, s)+\ldots
\end{aligned}
$$

Аналогично для $U(x, \mathrm{~s})$ выразим (20)

$$
U(X, s)=K i_{q L}(s) M_{1}(X, s)+K i_{m L}(s) M_{2}(X, s)
$$


и представим передаточные функции $M_{1}$ и $M_{2}$ также в виде разложений в ряд по малому параметру $s(21),(22)$

$$
\begin{aligned}
& M_{1}(X, s) \simeq \chi_{0}(X, s)+s \chi_{1}(X, s)+s^{2} \chi_{2}(X, s)+\ldots \\
& M_{2}(X, s) \simeq v_{0}(X, s)+s v_{1}(X, s)+s^{2} v_{2}(X, s)+\ldots
\end{aligned}
$$

Подставляя данные разложения в основное уравнение (9) и приравнивая коэффициенты при одинаковых степенях s, имеем следующую систему для определения сомножителей $\varphi_{0}, \psi_{0}, \varphi_{1}, \psi_{1} \ldots$

$$
\begin{gathered}
\varphi_{0}^{\prime \prime}=0 ; \\
\varphi_{1}^{\prime \prime}-Y_{1} \varphi_{0}=0 ; \\
\psi_{0}^{\prime \prime}=0 ; \\
\psi_{1}^{\prime \prime}-Y_{1} \psi_{0}=0 .
\end{gathered}
$$

Данные системы уравнений второго порядка при интегрировании требуют задания двух граничных условий для нахождения двух констант интегрирования. Первая константа найдётся из условия симметрии.

$$
\begin{aligned}
& \varphi_{0}^{\prime}(0, s)=0 ; \\
& \varphi_{1}^{\prime}(0, s)=0 ; \\
& \psi_{0}^{\prime}(0, s)=0 ; \\
& \psi_{1}^{\prime}(0, s)=0 .
\end{aligned}
$$

Вторую константу найдем из следующего интегрального условия:

$$
\begin{gathered}
\int_{0}^{1} s \varphi_{0} d x=1 ; \\
\int_{0}^{1} \varphi_{0}^{\prime \prime} d x=Y_{1} \int_{0}^{1} \varphi_{0} d x \\
\int_{0}^{1} s \psi_{0} d x=1 ; \\
\int_{0}^{1} \psi_{1}^{\prime \prime} d x=Y_{1} \int_{0}^{1} \psi_{0} d x .
\end{gathered}
$$

Решая систему (23)-(26) с учетом (27)-(30) и (31)-(34), имеем асимптотику решения с учетом двух членов разложения:

$$
\begin{aligned}
\Theta(X, F o) & \simeq \int_{0}^{F o} K i_{q}(\eta) d \eta-K i_{q}(F o) \times Y_{1}\left(\frac{X^{2}}{2}-1\right)+ \\
+(1-\varepsilon) & \times P n L u K o \int_{0}^{F o} K i_{m}(\eta) d \eta+K i_{m}(F o) \times \\
& \times Y_{1}\left(\frac{X^{2}}{2}-(1-\varepsilon) L u K o\right)+\ldots
\end{aligned}
$$

Используя известную для стадии падающей скорости сушки связь между теплообменным и массообменным критерием Кирпичева (36)

$$
K i_{q}(F o)=K i_{m}(F o) L u K o(1+R b),
$$

где $K i_{q}=\frac{q_{n}(t) R v}{\lambda T_{c}}, K i_{m}=\frac{j_{n}(t) R v}{a_{m} \rho_{0} \bar{u}_{0}}$.
Представим (35) в следующем виде:

$$
\Theta(X, F o)-\bar{\Theta}_{0} \simeq z_{1} \int_{0}^{F o} K i_{q}(\eta) d \eta+z_{2} K i_{q}(F o)+\ldots
$$

где

$$
z_{1}=\left[1+\frac{(1-\varepsilon) P n}{1+R b}\right], z_{2}=Y_{1}\left[1-\frac{X^{2}}{2}+\frac{\frac{X^{2}}{2}-(1-\varepsilon) L u K o}{L u K o(1+R b)}\right]
$$

Для расчета по (37) вначале необходимо определить температуру на поверхности при $x=1 \Theta(1, F o)=\Theta_{w}$. Полагая в (37) $x=1$, получим интегральное уравнение Вольтерра II рода (38)

$$
\begin{gathered}
\Theta_{w} \simeq z_{1} \int_{0}^{\mathrm{Fo}}\left[K i_{w}-S k\left(\Theta_{w}^{4}-1\right)-B i\left(\Theta_{w}-1\right)\right] d \eta+ \\
+z_{2}\left[K i_{w}-S k\left(\Theta_{w}^{4}-1\right)-B i\left(\Theta_{w}-1\right)\right]+\ldots \\
\int_{\Theta_{w}^{*}}^{\Theta_{w}} \frac{1+z_{2} B i+4 z_{2} \Theta_{w}^{3}}{K i_{w}-S k\left(\Theta_{w}^{4}-1\right)-B i\left(\Theta_{w}-1\right)} d \Theta_{w}=z_{1}\left(F o-F O_{*}\right) .
\end{gathered}
$$

Раскрывая интеграл (39), можно записать окончательное решение для $\Theta_{w}$ в элементарных функциях (40)

$$
z_{1}\left(F o-F O_{*}\right)=F_{1}\left(\Theta_{w}\right)-F_{1}\left(\Theta_{w}^{*}\right),
$$

где $F_{1}$ имеет вид

$$
\begin{aligned}
& F_{1}\left(\Theta_{w}\right)=\frac{1}{4(K i+S k+B i)^{\frac{3}{4}} S k} \times \\
& \times\left(\begin{array}{l}
2 S k^{\frac{3}{4}}\left(1+B i \cdot z_{2}\right) \operatorname{Arctan}\left[\frac{S k^{\frac{1}{4}} \Theta_{w}}{(K i+S k+B i)^{\frac{1}{4}}}\right]- \\
-S k^{\frac{3}{4}}\left(1+B i \cdot z_{2}\right) \times \\
\times\left(\begin{array}{l}
\log \left[(K i+S k+B i)^{\frac{1}{4}}-S k^{\frac{1}{4}} \Theta_{w}\right]- \\
-\log \left[(K i+S k+B i)^{\frac{1}{4}}+S k^{\frac{1}{4}} \Theta_{w}\right]
\end{array}\right)- \\
-4(K i+S k+B i)^{\frac{3}{4}} z_{2} \log \left[(K i+S k+B i)-S k \Theta_{w}{ }^{4}\right]
\end{array}\right)
\end{aligned}
$$

Зная теперь температуру поверхности можно рассчитать и полное распределение температуры по сечению пластины по зависимости (37).

Для оценки влагосодержания необходимо знать зависимость между массообменным критерием Кирпичева и временем. Ее получение вызывает определенные трудности. Чтобы обойти их, нами принят следующий подход. Поле температур в третьей части, как и в двух предыдущих частях статьи, исследуется более детально. Влагосодержание изучается менее подробно, для чего привлекаются наиболее апробированные практикой экспериментально-корреляционные связи между определяющими параметрами. В результате имеем (41)

$$
K i_{m}(t)=\frac{l^{2}}{a_{m}\left(W_{0}-W_{\mathrm{p}}\right)} \frac{d w}{d t}=\frac{\chi N l^{2}}{a_{m}} \frac{W-W_{\mathrm{p}}}{W_{0}-W_{\mathrm{p}}},
$$


где $\chi$ - относительный коэффициент сушки, $1 / \% ; N-$ скорость сушки, \%/c; $W$ - текущее влагосодержание, $\% ; W_{0}$ - начальное влагосодержание, $\% ; W_{\mathrm{p}}-$ равновесное влагосодержание, \%.

Переход от II стадии к III фиксируется достижением пластиной критического влагосодержания $W_{\text {кр }}$ равенством скоростей сушки, то есть (42)

$$
\left.\frac{d \bar{w}}{d t}\right|_{I I}=\left.\frac{d \bar{w}}{d t}\right|_{I I I} .
$$

В итоге имеем выражение для критического влагосодержания (43)

$$
\bar{W}_{\text {кр }}=\bar{W}-W_{\mathrm{p}}+\left(\frac{A}{1-\beta}\right)^{1 / \mathrm{\kappa}},
$$

где $A, \beta$, к - коэффициенты сушки; $\bar{W}, W_{\mathrm{p}}$ - среднее текущее и равновесное влагосодержание, кг/кг.

Равновесие влагосодержания пластины, соответствующее параметрам окружающей среды, определяется по известному уравнению Ленгмюра (44).

$$
W_{\mathrm{p}}=\left[D-E\left(T_{c}-273\right)\right]\left(\frac{\varphi}{F+\varphi}\right)^{0,5} .
$$

Здесь $D, E, F$ - постоянные, зависящие от материала; $\varphi$ - относительная влажность среды.

Для III стадии скорость сушки определяется по формуле Г.К. Филоненко [21] (45).

\section{СПИСОК ЛИТЕРАТУРЫ}

1. Коновалов Н.П. Технология деструкции бурых углей методом нагрева энергией сверхвысоких частот. - Иркутск: Изд-во ИрГТУ, 2000. - 92 с.

2. Three-dimensional simulation of microwave heating coal sample with varying parameters / Yi-du Hong, Bai-quan Lin, He Li, Huaming Dai, Chuan-jie Zhu, HaoYao // Applied Thermal Engineering. - 2016. - V. 93. - P. 1145-1154.

3. Sensitivity analysis on the microwave heating of coal: A coupled electromagnetic and heat transfer model / Baiquan Lin, He Li, Zhongwei Chen, Chunshan Zheng, Yidu Hong, ZhengWang // Applied Thermal Engineering. - 2017. - V. 126. - P. 949-962.

4. Meda V., Orsat V., Raghavan V. Microwave heating and the dielectric properties of foods. // Woodhead Publishing Series in Food Science, Technology and Nutrition. - 2017. - P. 23-43.

5. Microwave drying performance of single-particle coal slime and energy consumption analyses / Zhanlong Song, Chuanming Jing, Liansheng Yao, Xiqiang Zhao, Wenlong Wang, Yanpeng Mao, Chunyuan Ma // Fuel Processing Technology. - 2016. - V. 143. P. 69-78.

6. Faisal Mushtaq, Ramli Mat, Farid Nasir Ani. Fuel production from microwave assisted pyrolysis of coal with carbon surfaces // Energy Conversion and Management. - 2016. - V. 110. - P. 142-153.

7. Drying behavior of lignite under microwave heating / Zhanlong Song, Liansheng Yao, Chuanming Jing, Xiqiang Zhao, Wenlong Wang \& Chunyuan Ma // Drying Technology. - 2017. - V. 35. - P. 433-443.

8. Radha Raman Mishra, Apurbba Kumar Sharma. Microwavematerial interaction phenomena: heating mechanisms, challenges and opportunities in material processing // Composites Part A: Applied Science and Manufacturing. - 2016. - V. 81. - P. 78-97.

9. Temperature rise characteristics of Zhun Dong coal during microwave pyrolysis / Huan-Peng Liu, Tai-Ping Chen, Yang Li, Zhen-Yu Song, Shao-Wei Wang, Shao-Hua Wu // Fuel Processing Technology. - 2016. - V. 148. - P. 317-323.

10. Хайдурова А.А., Федчишин В.В., Коновалов Н.П. Микроволновая сушка бурых углей и повышение их технологических характеристик // Известия высших учебных заведений. Проблемы энергетики. - 2010. - С. 31-35.

$$
\left.\frac{d \bar{w}}{d t}\right|_{I I I}=\left.\frac{d \bar{w}}{d t}\right|_{I I} \frac{\left(\bar{W}-W_{\mathrm{p}}\right)^{\mathrm{K}}}{A+\beta\left(\bar{W}-W_{\mathrm{p}}\right)} .
$$

\section{Заключение}

В режиме падающей скорости сушки температура и влагосодержание подсушиваемого тела определяются взаимосвязью внутреннего и внешнего тепломассообмена. В рассматриваемом в статье случае это требует интегрирования нелинейной системы поставленных уравнений. Получить расчетные соотношения приближенно-аналитического характера удалось построением асимптотических приближений для малых и больших чисел Фурье. При этом дополнительно использована связь между теплообменным и массообменным критерием Кирпичева через критерий Ребиндера. В итоге тепловая часть задачи доведена до интегралов, которые выражаются через элементарные функции. В работе процесс переноса тепла учитывался более детально, чем массы. Сама скорость сушки определена по аппроксимационной формуле Г.К. Филоненко, в которой равновесное влагосодержание определено по уравнению Лентмюра.

Исследование и постановка задачи для стадии падающей скорости сушки выполнены в рамках государственного задания ИТ СО РАН, поиск приближенных аналитических решений выполнен за счет гранта РФФИ (№ 17-08-00752).

11. Методы и системы для повышения свойств твердого топлива: пат. USA 2007/0295.590.

12. Effect of microwave irradiation on the preparation of iron oxide/arenaceous clay sorbent for hot coal gas desulfurization $\mathrm{Yu}$ Feng, Tianqi $\mathrm{Hu}$, Mengmeng Wu, Ju Shangguan, Huilin Fan, Jie Mi // Fuel Processing Technology. - 2016. - V. 148. - P. 35-42.

13. Experimental study on the petrophysical variation of different rank coals with microwave treatment / He Li, Baiquan Lin, Wei Yang, Chunshan Zheng, Yidu Hong, Yabin Gao, Tong Liu, Shiliang Wu // International Journal of Coal Geology. - 2016. - V. 154-155. P. 82-91.

14. Activated carbon obtained from sapelli wood sawdust by microwave heating for o-cresol adsorption. / P.S. Thue, G.S. dos Reis, E.C. Lima, J.M. Sieliechi, G.L. Dotto, A.G.N. Wamba, S.L.P. Dias, F.A. Pavan // Research on Chemical Intermediates. February 2017. - V. 43. - Iss. 2. - P. 1063-1087.

15. Effect of microwave irradiation on petrophysical characterization of coals / Hong Yi-du, Lin Bai-quan, Zhu Chuan-jie, Li He // Applied Thermal Engineering. - 5 June 2016. - V. 102. - P. 1109-1125.

16. Jing Sun, Wenlong Wang, Qinyan Yue. Review on MicrowaveMatter Interaction Fundamentals and Efficient MicrowaveAssociated Heating Strategies // Materials. - 2016. - P. 231. DOI: 10.3390/ma9040231.

17. Rajasekhar Reddy B., Vinu R. Microwave assisted pyrolysis of Indian and Indonesian coals and product characterization // Fuel Processing Technology. - 2016. - V. 154. - P. 96-103.

18. Microwave absorption characteristics of anthracite during pyrolysis / Zhiwei Peng, Xiaolong Lin, Xuejiao Wu, Jiann-Yang Hwang, Byoung-Gon Kim, Yuanbo Zhang, Guanghui Li, Tao Jiang // Fuel Processing Technology. - 2016. - V. 150. - P. 58-63.

19. Лыков А.В. Теория теплопроводности. - М.: Изд-во «Высшая школа» 1967. - $600 \mathrm{c}$

20. Лыков А.В. Теория сушки. - М.: Изд-во «Энергия», 1968. $471 \mathrm{c}$.

21. Филоненко Г.К. Кинетика сушильных процессов. - М.: Оборониздат, 1939. - $140 \mathrm{c}$. 


\section{Информация об авторах}

Саломатов В.В., доктор технических наук, профессор кафедры физики неравновесных процессов Новосибирского государственного университета; главный научный сотрудник института теплофизики им. С.С. Кутателадзе СО РАН.

Карелин В.A., аспирант кафедры физики неравновесных процессов Новосибирского государственного университета; инженер-исследователь Института теплофизики им. С.С. Кутателадзе СО РАН.

Саломатов B.B., кандидат физико-математических наук, ведущий программист Института теплофизики им. С.С. Кутателадзе СО РАН. 


\title{
UDC 621.311 \\ ELECTROMAGNETIC DRYING OF WET MATERIALS WITH A SMALL DEPTH OF PENETRATION OF MICROWAVE RADIATION IN THE CONDITIONS OF HEAT REMOVAL BY RADIATION AND CONVECTION. III. STAGE OF FALLING DRYING RATE
}

\author{
Vladimir V. Salomatov ${ }^{1,2}$, \\ salomatov.vv@mail.ru \\ Vadim A. Karelin 1,2 , \\ vad2hen@mail.ru \\ Vasiliy V. Salomatov², \\ vvsalomatov @mail.ru \\ ${ }^{1}$ Novosibirsk State University, \\ 1, Pirogov street, Novosibirsk, 630090, Russia. \\ 2 S.S. Kutateladze Institute of Thermophysics SB RAS, \\ 1, Academician Lavrentiev avenue, Novosibirsk, 630090, Russia.
}

The relevance of the research is dictated by the need to develop mathematical models of microwave heating and MV-drying of wet materials to obtain technologically optimal and cost-effective modes. This publication is a continuation of the articles of the same authors in The Bulletin of the Tomsk Polytechnic University. Geo Assets Engineering, in which using the mathematical modeling, the authors have studied in details the process at the first and second stages of drying - the heating stage, when heat exchange between the surface of a wet body and the environment occurs due to radiation and convection, and microwave energy is absorbed by the surface layer owing to its small penetration depth, as well as the stage of constant drying rate. The authors constructed the asymptotic solutions of this nonlinear problem for small and large values of dimensionless time, which are demanded by engineering practice, both for parametric analysis and for performing operational calculations. The approach associated with the consideration of the third stage - the stage of the falling drying rate, is based on determination of the basic drying equation, which provides the relationship between heat transfer and moisture exchange using the Rebinder criterion.

The aim of the research is to state the problem of the third stage of microwave drying of a wet material - the stage of a falling drying rate, and to implement a theoretical solution to determine temperature field distribution over the layer thickness and the drying rate.

The object of the research is a flat layer of wet material - coal, sand, wood, etc. capillary-porous arrays, which are affected by microwave radiation. Such materials have a high dielectric constant and, as a result, very effectively absorb microwave radiation, which is almost $100 \%$ converted to thermal energy.

The research methods are associated with mathematical modeling, which are based on the equations of Maxwell's electrodynamics and heat and moisture transfer by A.V. Lykov. In this article, when assessing the parameters of heat and moisture transfer, heat transfer accounting is conducted in more details than mass transfer. One of the features of this problem is the consideration of materials with a small depth of absorption, whereby the source term in the system of equations for heating is in the boundary condition.

The temperature and moisture content of the body to be dried were determined in the mode of the falling drying speed, the calculated analytical ratios for small and large parameters of time were obtained.

\section{Key words:}

Microwave radiation, electromagnetic drying, capillary-porous array, heat radiation, convection, A.V. Lykov heat-moisture transfer equation, stage of falling drying speed.

The statement of the problem and the solution for the stage of falling drying rate were performed within the framework of the state task IT SB RAS, the search for approximate analytical solutions was done with the support of the Russian Foundation for Basic Research, grant No. 17-08-00752.

\section{REFERENCES}

1. Konovalov N.P. Tekhnologiya destruktsii burykh ugley metodom nagreva energiey sverkhvysokikh chastot [Technology of of brown coal destruction by the method of heating with energy of ultrahigh frequencies]. Irkutsk, IrSTU Publ. house, 2000. 92 p.

2. Yi-du Hong, Bai-quan Lin, He Li, Hua-ming Dai, Chuan-jie Zhu, HaoYao. Three-dimensional simulation of microwave heating coal sample with varying parameters. Applied Thermal Engineering, 25 January 2016, vol. 93, pp. 1145-1154.

3. Baiquan Lin, He Li, Zhongwei Chen, Chunshan Zheng, Yidu Hong, Zheng Wang. Sensitivity analysis on the microwave heating of coal: a coupled electromagnetic and heat transfer model. Applied Thermal Engineering, 5 November 2017, vol. 126, pp. 949-962.

4. Meda V., Orsat V., Raghavan V. Microwave heating and the dielectric properties of foods. Woodhead Publishing Series in Food Science, Technology and Nutrition, 2017, pp. 23-43.
5. Zhanlong Song, Chuanming Jing, Liansheng Yao, Xiqiang Zhao, Wenlong Wang, Yanpeng Mao, Chunyuan Ma. Microwave drying performance of single-particle coal slime and energy consumption analyses. Fuel Processing Technology, March 2016, vol. 143, pp. 69-78.

6. Faisal Mushtaq, Ramli Mat, Farid Nasir Ani. Fuel production from microwave assisted pyrolysis of coal with carbon surfaces. Energy Conversion and Management, 15 February 2016, vol. 110, pp. 142-153.

7. Zhanlong Song, Liansheng Yao, Chuanming Jing, Xiqiang Zhao, Wenlong Wang, Chunyuan Ma. Drying behavior of lignite under microwave heating. Drying Technology, 31 Jan 2017, vol. 35, pp. 433-443.

8. Radha Raman Mishra, Apurbba Kumar Sharma. Microwavematerial interaction phenomena: Heating mechanisms, challenges and opportunities in material processing. Composites Part A: Applied Science and Manufacturing, February 2016, vol. 81, pp. 78-97. 
9. Huan-Peng Liu, Tai-Ping Chen, Yang Li, Zhen-Yu Song, ShaoWei Wang, Shao-Hua Wu. Temperature rise characteristics of Zhun Dong coal during microwave pyrolysis. Fuel Processing Technology, July 2016, vol. 148, pp. 317-323.

10. Khaidurova A.A., Fedchishin V.V., Konovalov N.P. Microwave drying of brown coals and increasing their technological characteristics. Izv. Universities. Problems of energy, 2010, pp. 31-35. In Rus.

11. Methods and systems for improving the properties of solid fuels. Patent USA 0295.590, 2007.

12. Yu Feng, Tianqi Hu, Mengmeng Wu, Ju Shangguan, Huilin Fan, Jie Mi. Effect of microwave irradiation on the preparation of iron oxide/arenaceous clay sorbent for hot coal gas desulfurization. Fuel Processing Technology, July 2016, vol. 148, pp. 35-42.

13. He Li, Baiquan Lin, Wei Yang, Chunshan Zheng, Yidu Hong, Yabin Gao, Tong Liu, Shiliang Wu. Experimental study on the petrophysical variation of different rank coals with microwave treatment. International Journal of Coal Geology, 15 January 2016, vol. $154-155$, pp. 82-91.

14. Thue P.S., Dos Reis G.S., Lima E.C., Sieliechi J.M., Dotto G.L., Wamba A.G.N., Dias S.L.P., Pavan F.A. Activated carbon obtained from sapelli wood sawdust by microwave heating for ocresol adsorption. Research on Chemical Intermediates, February 2017, vol. 43, Iss. 2, pp. 1063-1087.

\section{Information about the authors}

Vladimir V. Salomatov, Dr. Sc., professor, Novosibirsk State University; senior researcher, S.S. Kutateladze Institute of Thermophysics SB RAS.

Vadim A. Karelin, postgraduate, Novosibirsk State University; engineer, SS. Kutateladze Institute of Thermophysics SB RAS.

Vasiliy V. Salomatov, Cand. Sc., leading programmer, S.S. Kutateladze Institute of Thermophysics SB RAS.
15. Hong Yi-du, Lin Bai-quan, Zhu Chuan-jie, Li He. Effect of microwave irradiation on petrophysical characterization of coals. Applied Thermal Engineering, 2016, vol. 102, pp. 1109-1125.

16. Jing Sun, Wenlong Wang, Qinyan Yue. Review on MicrowaveMatter Interaction Fundamentals and Efficient MicrowaveAssociated Heating Strategies. Materials, 20166 pp. 231. DOI: 10.3390/ma904023.

17. Rajasekhar Reddy B., Vinu R. Microwave assisted pyrolysis of Indian and Indonesian coals and product characterization. Fuel Processing Technology, 15 December 2016, vol. 154, pp. 96-103.

18. Zhiwei Peng, Xiaolong Lin, Xuejiao Wu, Jiann-Yang Hwang, Byoung-Gon Kim, Yuanbo Zhang, Guanghui Li, Tao Jiang. Microwave absorption characteristics of anthracite during pyrolysis. Fuel Processing Technology, September 2016, vol. 150, pp. 58-63.

19. Lykov A.V. Teoriya teploprovodnosti [Theory of heat conductivity]. Moscow, Vysshaya shkola Publ., 1967. $600 \mathrm{p}$.

20. Lykov A.V. Teoriya sushki [The theory of drying]. Moscow, Energiya Publ., 1968. 471 p.

21. Filonenko G.K. Kinetika sushilnykh protsessov [Kinetics of drying processes]. Moscow, Oboronizdat Publ., 1939. 140 p.

Received: 12 March 2020. 